\title{
MiR-98 modulates macrophage polarization and suppresses the effects of tumor-associated macrophages on promoting invasion and epithelial- mesenchymal transition of hepatocellular carcinoma
}

\author{
Lei Li ${ }^{1}$, Pengfei Sun ${ }^{1}$, Chengsheng Zhang ${ }^{1}$, Zongchao $\mathrm{Li}^{1}$, Kai Cui ${ }^{1}$ and Wuyuan Zhou²*
}

\begin{abstract}
Background: Tumor-associated macrophages (TAMs) are generally recognized as a promoter of tumor progression. miR-98 has been shown to suppress the proliferation, migration, invasion and epithelial-mesenchymal transition (EMT) of hepatocellular carcinoma (HCC) cells. Here, we aim to investigate the role of miR-98-mediated macrophage polarization in HCC progression.

Methods: Human blood monocytes were isolated from healthy male donors and incubated with culture medium collected from HepG2 cells for 7 days. The phenotype of the macrophages was detected. The protein expression was detected by Western blot. Levels of cytokines secreted in culture medium were measured using the specific enzymelinked immunosorbent assay kits. To explore the role of miR-98 in HCC-conditioned TAMs, HCC cells HepG2 and SMMC7721 were cultured with conditioned medium from HCC-conditioned TAMs that had been transfected with miR-98 mimic/inhibitor. Cell proliferation, migration and invasion assays were performed.
\end{abstract}

Results: HCC-conditioned TAMs possessed M2-like phenotype, including increased protein expression of CD163 and

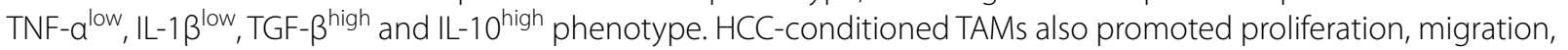
invasion and EMT of HepG2 and SMMC7721 cells. Furthermore, miR-98 modulated macrophage polarization from M2 to M1 in HCC-conditioned TAMs, as evidenced by the alteration of M1- or M2-related cytokines. Moreover, miR-98 mimic significantly suppressed the HCC-conditioned TAMs-mediated promotion of cell migration, invasion and EMT in HepG2 and SMMC7721 cells compared with negative control, whereas miR-98 inhibitor exerted reversed effects.

Conclusions: miR-98 may play a vital role in regulating macrophage polarization, thereby suppressing the TAMsmediated promotion of invasion and EMT in HCC.

Keywords: miR-98, Macrophage polarization, Tumor-associated macrophages, Epithelial-mesenchymal transition, Hepatocellular carcinoma

\footnotetext{
*Correspondence: 15564100001@163.com

2 Department of Hepatobiliary and Pancreatic Surgery, Xuzhou Cancer

Hospital, No. 131 Huancheng Road, Gulou District, Xuzhou 221000,

Jiangsu, China

Full list of author information is available at the end of the article
}

(c) The Author(s) 2018. This article is distributed under the terms of the Creative Commons Attribution 4.0 International License (http://creativecommons.org/licenses/by/4.0/), which permits unrestricted use, distribution, and reproduction in any medium, provided you give appropriate credit to the original author(s) and the source, provide a link to the Creative Commons license, and indicate if changes were made. The Creative Commons Public Domain Dedication waiver (http://creativecommons.org/ publicdomain/zero/1.0/) applies to the data made available in this article, unless otherwise stated. 


\section{Background}

Hepatocellular carcinoma ( $\mathrm{HCC})$ is one of the leading causes of cancer-related death worldwide, with 1 million deaths a year $[1,2]$. Therefore, understanding of the molecular mechanisms underlying HCC is important for the development of effective treatment strategies. The tumor microenvironment is composed of cancer tissue and the surrounding stromal cells, and provides opportunity for reciprocal interactions among inflammatory cells, cancer cells and microcapillary vessels [3]. Interestingly, HCC is one of inflammation-related cancers, as the chronic inflammatory state appears to be necessary for the initiation and development of liver cancer [4].

Macrophages, highly plastic cells, can be polarized to achieve a spectrum of functional phenotypes. In response to a variety of microenvironmental stimuli [5]. Pro-inflammatory M1 macrophages show tumoricidal activity and promote $\mathrm{T}$ helper (Th) 1 responses, whereas M2 macrophages display regulatory functions in tissue repair and remodeling, and promote Th2 immune responses [6]. In addition, M1 macrophages can enhance cell recruitment to the inflammatory focus by secreting tumor necrosis factor (TNF)- $\alpha$, interleukin (IL)-1 $\beta$, and nitric oxide. Furthermore, M2 macrophages enhance fungal phagocytosis and secrete pro-resolution substances including fibronectin, IL-10, transforming growth factor (TGF)- $\beta$, and metalloproteases [7]. Tumor-associated macrophages (TAMs) mostly show an M2-like phenotype [8]. TAMs are key orchestrators of the tumor microenvironment directly affecting neoplastic cell growth, neoangiogenesis, and extracellular matrix remodeling [9]. Indeed, increased infiltration of TAMs has been associated with poor prognosis and worse pathological characteristics in several cancers, including breast cancer, colon cancer, bladder cancer, prostate carcinoma, and also HCC $[3,10-12]$.

MicroRNAs (miRNAs) can regulate $30-90 \%$ of human genes and play important roles in cell growth, activation, apoptosis and differentiation [3]. miR-98, belongs to the let-7 family, acts as an oncogene or tumor suppressor in some human cancers $[13,14]$. miR-98 has been shown to inhibit HCC proliferation via targeting enhancer of zeste homolog-2 (EZH2) and suppressing Wnt/ $\beta$-catenin signaling pathway [15]. Recently, Lin-28B was shown to promote tumor formation and invasion in $\mathrm{HCC}$ through coordinated repression of the let-7/miR-98 family and induction of multiple oncogenic pathways [16]. Our previous study demonstrated that miR-98 played a suppressive role in the proliferation, migration, invasion and epithelial-mesenchymal transition (EMT) of HCC cells via targeting Sal-like protein 4 [17]. Recent studies have shown that let-7b, another member of the let-7 family, was up-regulated in prostatic TAMs and modulated macrophage polarization; and the decreased expression of let-7b inhibited the pro-angiogenic effect of TAMs and their capacity to enhance prostate carcinoma cell motility [3]. Therefore, we hypothesized that miR-98, another member of the let-7 family, may also modulate macrophage polarization and affect the effects of TAMs on the invasion and EMT of HCC. This study investigated the effects of miR-98 in regulating macrophage polarization and explored the role of miR-98-mediated macrophage polarization in HCC progression.

\section{Methods \\ Isolation and culture of human peripheral blood macrophages}

Blood monocytes were isolated from healthy donor buffy coats. Peripheral blood mononuclear cells (PBMCs) were isolated using a Ficoll (Solarbio Life Sciences, Beijing, China) density gradient. Monocytes were purified with anti-CD14 paramagnetic beads (Miltenyi Biotec, Auburn, CA), according to the manufacturer's instruction. Non-adherent cells were removed, and the purity of monocytes (>95\%) was determined by flow cytometric analysis. $\mathrm{CD} 14^{+}$monocytes $\left(5 \times 10^{5} \mathrm{cells} / \mathrm{ml}\right)$ were cultured with RPMI 1640 (Sigma Co., St. Louis, Mo., USA), supplemented with $10 \%$ fetal bovine serum (FBS; Sigma Co., St. Louis, Mo., USA) and $50 \mathrm{ng} / \mathrm{ml}$ macrophage colony-stimulating factor (M-CSF, Sigma Co., St. Louis, Mo., USA) for 7 days. Half of culture medium was changed every 3 days, unless otherwise indicated. To obtain M0 cells, CD14 ${ }^{+}$monocytes were treated with serum-free medium for $48 \mathrm{~h}$. To polarize M1 macrophages, cells were stimulated overnight with $100 \mathrm{ng} / \mathrm{ml}$ lipopolysaccharides (LPS, Sigma Co., St. Louis, Mo., USA) and 100 ng/ml IFN- $\gamma$ (Sigma Co., St. Louis, Mo., USA). To polarize M2 macrophages, cells were stimulated overnight with $20 \mathrm{ng} / \mathrm{ml}$ IL-4 (Sigma Co., St. Louis, Mo., USA). TAMs of HCC were obtained by culturing monocytes isolated from PBMCs for 7 days in RPMI 1640 containing $10 \%$ FBS with $50 \%$ of conditioned medium from HepG2 cells. Conditioned medium was obtained from untreated HepG2 cells. M0, M1, M2 and TAMs cells were incubated for $48 \mathrm{~h}$ in serum-starved condition, and culture medium was harvested and clarified by centrifugation and used freshly. Written informed consent was obtained from all donors. The study was approved by the Ethics Committee of Shandong Cancer Hospital Affiliated to Shandong University.

\section{Quantification of cytokine levels}

Levels of cytokines including TNF- $\alpha$, IL- $1 \beta$, TGF- $\beta$ and IL-10 secreted in culture medium were measured using the specific enzyme-linked immunosorbent assay 
(ELISA) system kits (Abcam, Chicago, IL USA), according to the manufacturer's instructions.

\section{Cell culture}

Human HCC cell lines HepG2 and SMMC7721 were purchased from the Chinese Academy of Sciences (Shanghai, China). Cells were cultured in Dulbecco's modified Eagle's medium (DMEM, Life Technologies), supplemented with 10\% FBS, $100 \mathrm{IU} / \mathrm{ml}$ penicillin and $100 \mathrm{IU} /$ $\mathrm{ml}$ streptomycin. Cells were cultured at $37{ }^{\circ} \mathrm{C}$ in a humidified atmosphere with $5 \% \mathrm{CO}_{2}$. Cells were then incubated with culture medium of M0, M1, M2, TAMs cells or 1640 medium as control for $48 \mathrm{~h}$. The culture medium of cells was harvested and clarified by centrifugation and used freshly.

\section{MTT assay}

The 3-(4, 5-dimethylthiazal-2-yl)-2, 5-diphenyl-tetrazolium bromide (MTT) assay was used to examine cell proliferation as described in our previous study [17]. Briefly, cells in each group were plated at a density of $1 \times 10^{4}$ cells per well in 96-well plates. Cells were then incubated with MTT at a final concentration of $0.5 \mathrm{mg} / \mathrm{ml}$ for $4 \mathrm{~h}$ at $37^{\circ} \mathrm{C}$. After the removal of the medium, $150 \mathrm{mM}$ DMSO solutions were added to dissolve the formazan crystals. The absorbance was measured at $570 \mathrm{~nm}$ using a BioTek $^{\mathrm{TM}}$ ELX-800 ${ }^{\mathrm{TM}}$ Absorbance Microplate reader (Bio-Tek Instruments Inc., USA).

\section{Cell migration assay}

Wound healing assay was performed to evaluate the cell migratory capacity of HCC cells as described in our previous study [17]. In brief, cells were cultured to reach $70-80 \%$ confluence. Wounds of approximately $1 \mathrm{~mm}$ width were created with a plastic scriber, and cells were washed and incubated in a serum-free medium. At $24 \mathrm{~h}$ after wounding, cells were incubated in a medium supplemented with $10 \%$ FBS. After 48 h of culture, cells were fixed and observed under a microscope (original magnification, 100×; Olympus, Tokyo, Japan).

\section{Cell invasion assay}

Cell invasion assay was performed using Transwell chambers (BD Biosciences, Bedford, Massachusetts, USA) as described in our previous study [17]. Cells were pre-coated with Matrigel. Cell suspension containing $5 \times 10^{5}$ cells $/ \mathrm{ml}$ was prepared in serum-free media, and $300 \mu \mathrm{l}$ cell suspension was added into the upper chamber. Then, $500 \mu \mathrm{l}$ DMEM with $10 \%$ FBS was added into the lower chamber. Cells were incubated for $24 \mathrm{~h}$. Then, we used a cotton-tipped swab to carefully wipe out the cells that did not invade through the pores. The filters were fixed in $90 \%$ alcohol and stained by crystal violet, and observed under an inverted microscope (original magnification, 100×; Olympus, Tokyo, Japan).

\section{miR mimic and inhibitor}

The miR-98 mimic, mimic negative control (NC), miR98 inhibitor and inhibitor NC were purchased from Amspring (Changsha, China), and were transfected into M0, M1, M2 and TAMs cells by Lipefectamin-2000.

\section{Western blot}

Western blot was performed as described in our previous study [17]. Cells were lysed with ice-cold lysis buffer (50 mM Tris-HCl, pH6.8, $100 \mathrm{mM} 2-\mathrm{ME}, 2 \% \mathrm{w} / \mathrm{v}$ SDS, $10 \%$ glycerol). After centrifugation at $20,000 \times g$ for $10 \mathrm{~min}$ at $4{ }^{\circ} \mathrm{C}$, proteins in the supernatants were quantified and separated with 10\% SDS-PAGE. Then, proteins were transferred onto a polyvinylidene difluoride (PVDF) membrane (Amersham Bioscience, Buckinghamshire, USA), which was then incubated with PBS containing $5 \%$ milk overnight at $4{ }^{\circ} \mathrm{C}$. The PVDF membrane was incubated with rabbit anti-human primary antibodies: CD163 (1:1000, ab17051), E-cadherin (1:1000, ab15148), N-cadherin (1:1000, ab18203), Fibronectin (1:1000, ab2413), vimentin (1:1000, ab16700) and GAPDH (1:10,000, ab181602) (all from Abcam, Cambridge, MA, USA) at room temperature for $3 \mathrm{~h}$, respectively, and then incubated with mouse anti-rabbit secondary antibody $(1: 10,000$, ab99702, Abcam) at room temperature for 1 h. Super Signal West Pico Chemiluminescent Substrate Kit (Pierce, Rockford, IL, USA) was then used to detect signals, according to the manufacture's instruction. The relative protein expression was analyzed by Image-Pro plus software 6.0, represented as the density ratio versus GAPDH.

\section{RNA extraction and real-time reverse transcription PCR}

Total RNA was extracted using Invitrogen Trizol Reagent (Life Technologies Corporation). For miRNA quantification, 100 ng total RNA was reverse transcribed directly using stem-loop primers. Quantitative real-time PCR was performed using the SYBR Green PCR Master Mix (Tokara, Kyoto, Japan) in a final volume of $20 \mu \mathrm{l}$ on BioRAD CFX96 TM Real-Time System (Bio-Rad Laboratories, Inc., Hercules, CA, USA). The expression of miRNA was normalized to U6. Data are presented as relative quantification based on the calculation of $2^{-\Delta \Delta \mathrm{Ct}}$.

\section{Statistical analysis}

SPSS16.0 software was used for statistical analysis. All data were presented as mean \pm standard deviation (SD) of three independent experiments. The error bars in each figure represent $\mathrm{SD}$ of three independent experiments. One-way analysis of variance (ANOVA) 
was used for comparison. $\mathrm{P}<0.05$ was considered to indicate a statistically significant difference.

\section{Results}

\section{Characterization of HCC-conditioned TAMs}

Circulating monocytes can pass through the vascular endothelium to mature into macrophages in the peripheral tissues and are activated in various ways through endogenous and exogenous factors. To investigate whether exposure to HCC tumor microenvironment affected monocyte differentiation, human blood monocytes were incubated with culture medium collected from HepG2 cells for 7 days, and the phenotype of the macrophages were detected (Additional file 1: Figure S1). Data revealed that the protein expression of CD163, a marker for M2, was significantly decreased in M1 group compared with the M0 group (Fig. 1a). On the contrary, the protein expression of CD163 was significantly increased in M2 and TAM group compared with the M0 group. Next, we measured the expression of TNF- $\alpha$, IL- $1 \beta$, TGF- $\beta$ and IL-10, which have been used for phenotyping macrophages. Our results showed that both M2 macrophages and TAMs displayed TNF- $\alpha^{\text {low }}$, IL- $1 \beta^{\text {low }}$, TGF- $\beta^{\text {high }}$ and IL- $10^{\text {high }}$ phenotype (Fig. 1b-e). These data indicated that HCCconditioned TAMs possessed M2-like phenotype.
HCC-conditioned TAMs promote proliferation, migration, invasion and EMT of HepG2 and SMMC7721 cells in vitro Given that HCC-conditioned TAMs possessed M2-like phenotype, we next analyzed whether these TAMs possessed the M2-like effects on HepG2 cells in vitro. HepG2 cells were then incubated with culture medium of M0, M1, M2 and TAMs cells or 1640 medium as control for $48 \mathrm{~h}$. Subsequently, we evaluated the proliferative, migratory and invasive variation of HepG2 cells. We found that HepG2 cells incubated with TAMs showed a remarkable promotion in cell proliferation (Fig. 2a), invasion (Fig. 2b), and migration (Fig. 2c) compared with the control HepG2 cell group. Furthermore, the TAMs group showed decreased protein levels of E-cadherin but increased levels of $\mathrm{N}$-cadherin, fibronectin and vimentin, indicating that EMT was induced by TAMs (Fig. 2d). These effects were comparable to the results observed in HepG2 cells that incubated with culture medium of M2 macrophages. Also, similar results were observed in SMMC7721 cells (Fig. 3).

\section{miR-98 regulates macrophage polarization in HCC-conditioned TAMs}

Next, we detected the expression of miR-98 in M0, M1, M2, and TAMs. We found that the expression levels of miR-98 in M2 and TAMs were significantly decreased compared with the M0 group (Fig. 4a). After that, we transfected TAMs with miR-98 mimic, mimic NC, miR98 inhibitor and inhibitor $\mathrm{NC}$ to investigate whether

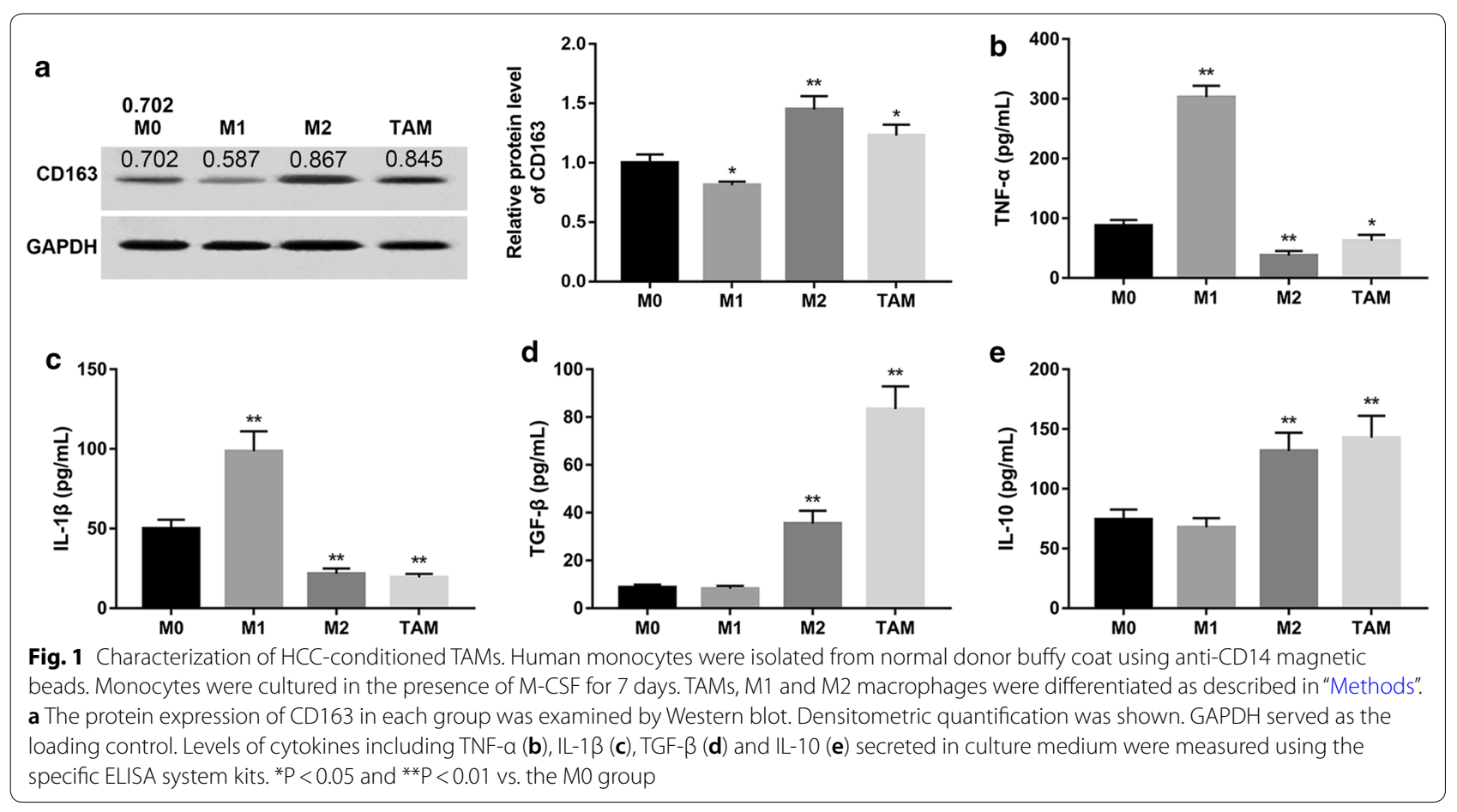



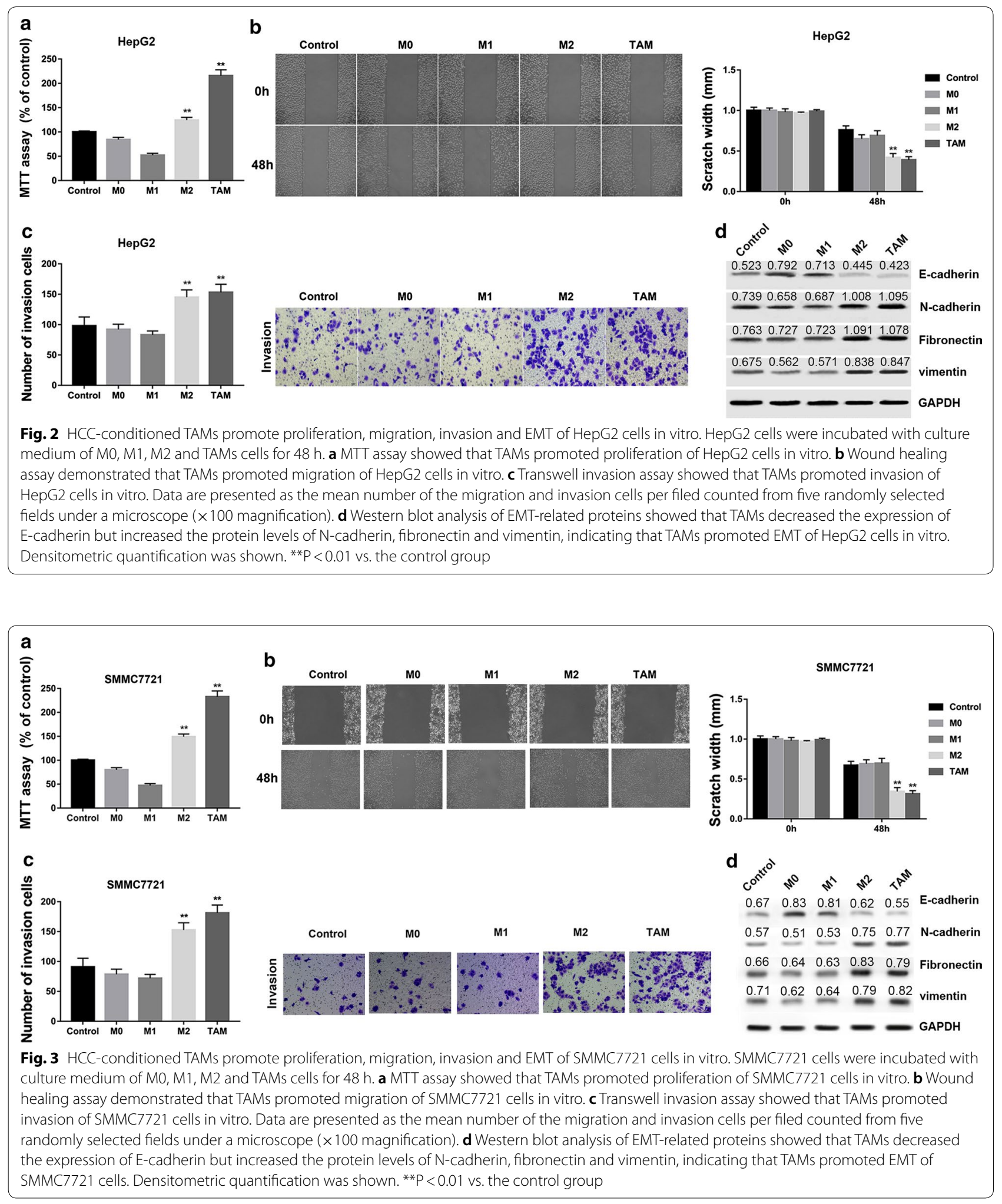


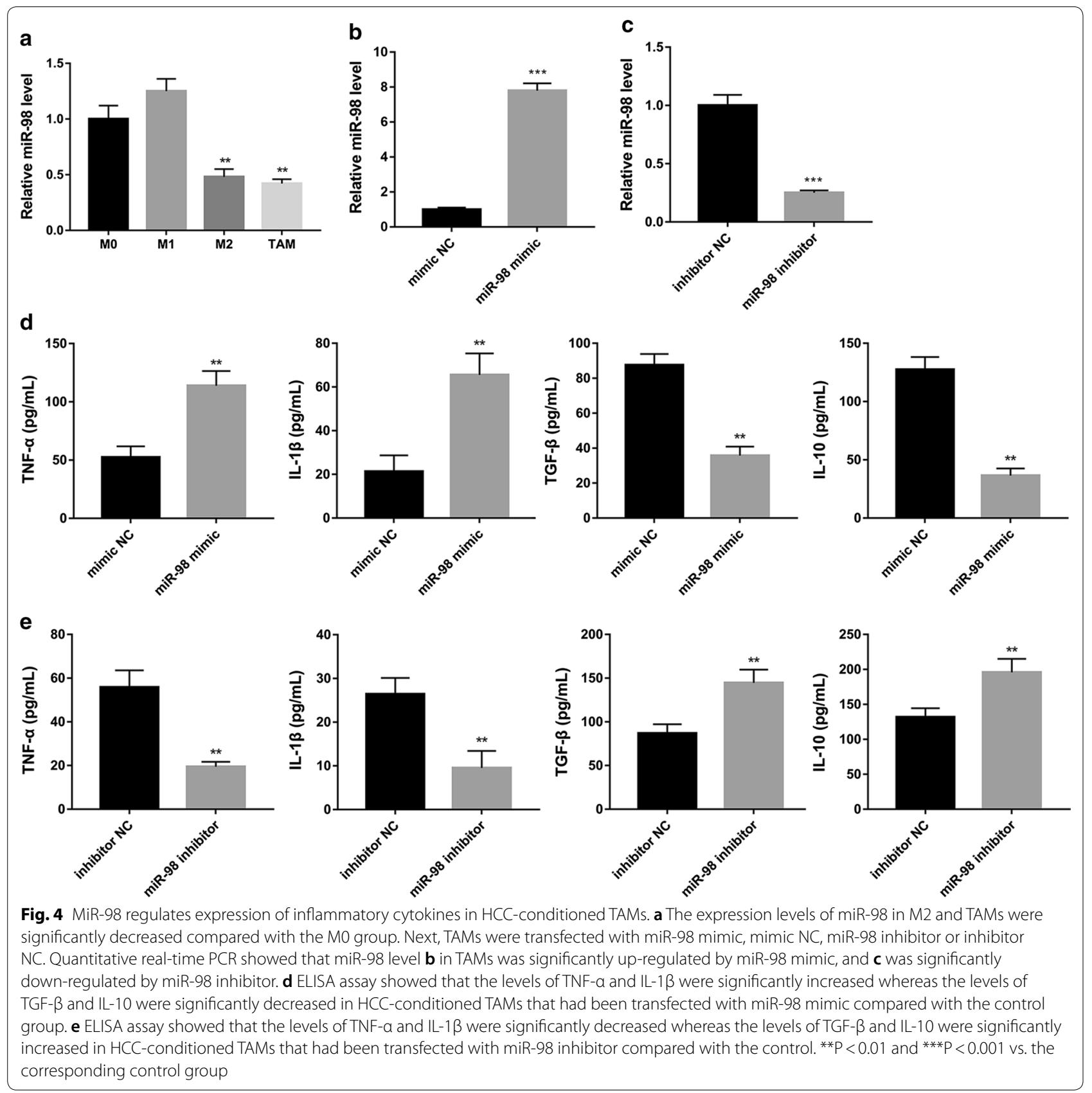

miR-98 regulated macrophage polarization in $\mathrm{HCC}$ conditioned TAMs. We found that miR-98 level was significantly up-regulated by miR-98 mimic (Fig. 4b) and significantly down-regulated by miR-98 inhibitor (Fig. 4c), compared with the corresponding controls. It has been reported that TAMs exhibited two different patterns of phenotype, M1 or M2, depending on their activation pathway [18]. M1 macrophages secrete TNF- $\alpha$ and IL- $1 \beta$, and M2 macrophages secrete TGF- $\beta$ and IL-10 [7]. Our results showed that, in the presence of
miR-98 mimic, the levels of TNF- $\alpha$ and IL- $1 \beta$ were significantly increased whereas the levels of TGF- $\beta$ and IL-10 were significantly decreased in HCC-conditioned TAMs (Fig. 4d). However, the levels of TNF- $\alpha$ and IL-1 $\beta$ were significantly decreased whereas the levels of TGF- $\beta$ and IL-10 were significantly increased in HCC-conditioned TAMs that transfected with miR-98 inhibitor compared with the control (Fig. 4e). These results indicated that miR-98 may modulate macrophage polarization from M2 to $\mathrm{M} 1$ in $\mathrm{HCC}$-conditioned TAMs. 


\section{HCC-conditioned TAMs treated with miR-98 regulate migration, invasion and EMT of HepG2 and SMMC7721 cells}

To clarify whether introduction of miR-98 mimic/inhibitor into TAMs could impair HepG2 and SMMC7721 cell growth, we incubated human HepG2 and SMMC7721 cells with culture medium from TAMs that had been transfected with miR-98 mimic/inhibitor or the corresponding controls. Data revealed that the capacity of HCC-conditioned TAMs to promote cell migration and invasion of HepG2 and SMMC7721 cells was significantly suppressed by miR-98 mimic but enhanced by miR-98 inhibitor compared with their corresponding control (Figs. 5a-d, and 6a-d). Furthermore, the capacity of HCC-conditioned TAMs to promote EMT of HepG2 and SMMC7721 cells was suppressed by miR-98 mimic but enhanced by miR-98 inhibitor compared with their corresponding control (Figs. 5e and 6e). These results suggested that miR-98 expression in HCC-conditioned TAMs played a critical role in regulating migration, invasion and EMT of HepG2 and SMMC7721 cells.

\section{Discussion}

TAMs have been associated with enhanced tumor progression, including cancer cell growth and immune suppression. In this study, human monocytes became HCC-conditioned TAMs after incubation with conditioned medium collected from HepG2 cell culture. The resultant TAMs displayed characteristics of M2-like macrophages, including increased protein expression of CD163, TNF- $\alpha^{\text {low }}$, IL- $1 \beta^{\text {low }}$, TGF- $\beta^{\text {high }}$ and IL- $10^{\text {high }}$ phenotype. The up-regulation of M2-associated CD163 in HCC-infiltrating macrophages has been recently demonstrated [19]. Moreover, an established M2 macrophage

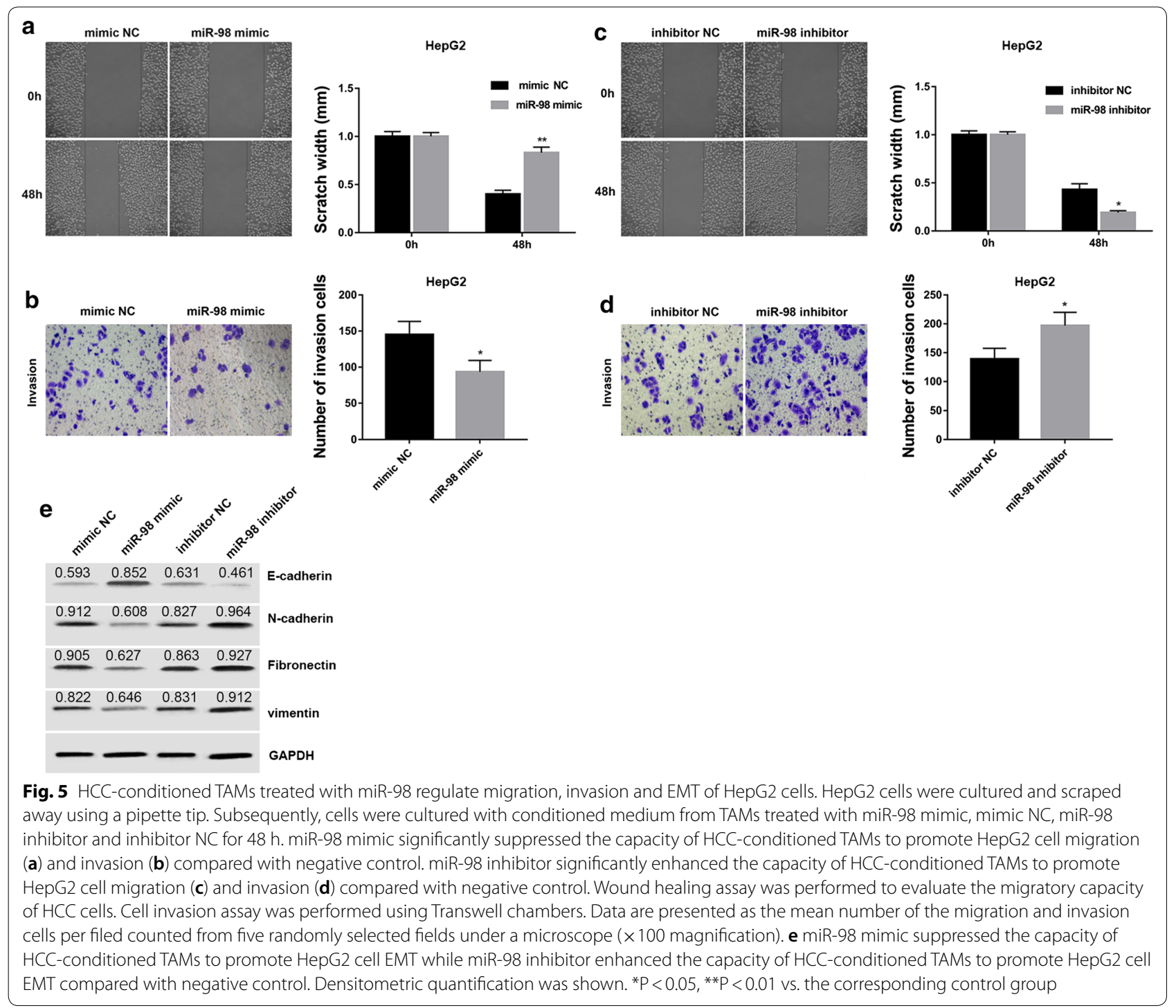




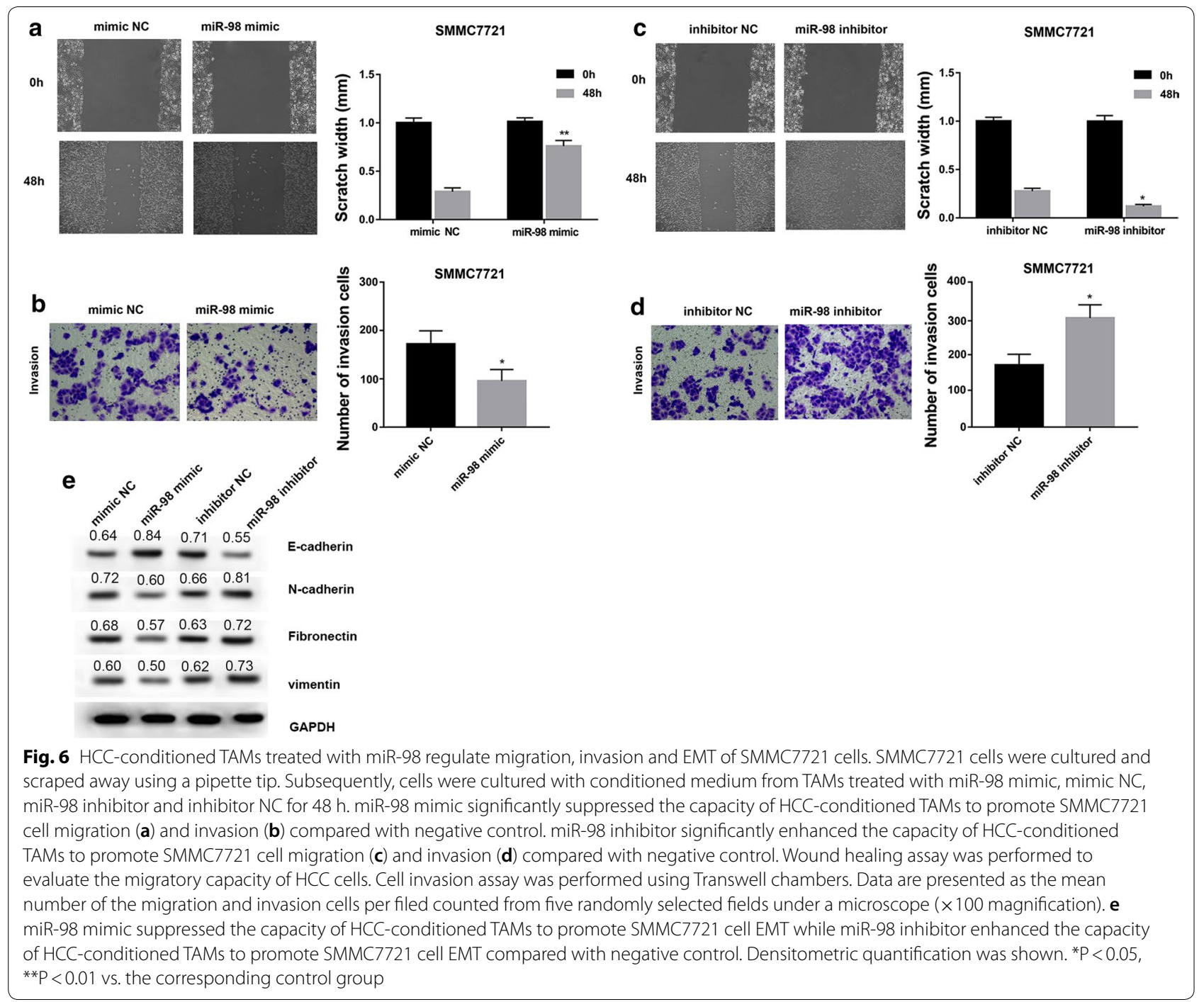

population has been associated with poor prognosis in HCC [20]. Here, we found that TAMs significantly enhanced cell migration, invasion, and EMT in HepG2 and SMMC7721 cells in vitro compared with the control macrophages. Our results indicate the important role of HCC-conditioned TAMs in HepG2 and SMMC7721 cells. However, inconsistent with the pro-tumor effects of TAMs in our study, other studies suggested that the infiltration of TAMs could protect HCC patients from recurrence and metastasis, and that high levels of proinflammatory molecules derived from tumor-infiltrating cells were associated with a better survival in HCC patients $[21,22]$. The contradictory results may be attributed to several factors such as the difference between in vivo and in vitro environment, number of samples, and different experimental procedures.

miRNAs are universal regulators of differentiation, activation and polarization of macrophages. For example,
Chaudhuri et al. [23] suggested that miR-125b is responsible for generating the activated nature of macrophages and potentiating the functional role of macrophages in inducing immune responses. Androulidaki et al. [24] indicated that the kinase Akt1 controls macrophage response to lipopolysaccharide by regulating microRNAs, such as let-7e, miR-181c, miR-155 and miR-125b. Kumar et al. [25] showed that let-7f, another member of the let-7 family, was over-expressed in tuberculosis-infected macrophages that induced TNF and IL- $1 \beta$ secretion. A recent study demonstrated that miRNA let-7b modulates macrophage polarization and the decreased expression of let-7b inhibits the pro-angiogenic effect of TAMs and their capacity to enhance prostate carcinoma cell motility [3]. However, the role of miR-98, another member of the let-7 family, in modulating macrophage polarization, has not yet been defined. Our results showed that miR-98 is expressed in HCC-conditioned TAMs at the lower level 
compared with M0 and M1 macrophages. Furthermore, miR-98 mimic significantly increased levels of TNF- $\alpha$ and IL-1 $\beta$ (secreted by M1 macrophages) but decreased levels of TGF- $\beta$ and IL-10 (secreted by M2 macrophages) in HCC-conditioned TAMs. In contrast, miR-98 inhibitor exerted the opposite effects on these cytokines. These results indicate that miR-98 regulates macrophage polarization from M2 to M1 in HCC-conditioned TAMs.

Some studies have demonstrated that miR-98 has suppressive effects on several cancers, such as oral squamous cell carcinoma, non-small-cell lung cancer, glioma, melanoma and HCC [17, 26-29]. A recent study demonstrated that the CCL18-mediated down-regulation of miR-98 enhanced the EMT of breast cancer cells, and thus promoted breast cancer metastasis [30]. In this study, to explore the role of miR-98 in HCC-conditioned TAMs, HepG2 and SMMC7721 cells were cultured with conditioned medium from TAMs that were treated with miR-98 mimic or miR-98 inhibitor for $48 \mathrm{~h}$. We found that miR-98 not only regulated expression of inflammatory cytokines in HCC-conditioned TAMs, but also suppressed the capacity of HCC-conditioned TAMs to promote HepG2 and SMMC7721 cell migration, invasion, and EMT. One plausible explanation might be that miR-98 regulates macrophage polarization from M2 to M1 in HCC-conditioned TAMs. Furthermore, a previous study demonstrated that the TAM-released cytokines and chemokines play a vital role in the initiation and progression of liver cancer and regulation of tumor growth, invasion, and metastasis [4]. Collectively, the miR-98-mediated macrophage polarization from M2 to M1 and regulation of cytokines may lead to the change in biological properties of TAMs.

It is well-established that miRNAs inhibit protein expression by binding to the 3'-untranslated region of target mRNAs, leading to transcriptional repression or degradation of the mRNA. By using TargetScan and PicTar, several HCC-related genes (such as IL-10, LIN28B, HMGA2) [16, 31] were identified as the potential targets of miR-98. Therefore, we speculate that these potential targets may involve in the miR-98-mediated regulation in macrophage polarization in HCC-conditioned TAMs and in the capacity of HCC-conditioned TAMs to regulate HCC cell migration and invasion, which needs our further investigation.

\section{Conclusions}

In conclusion, our findings showed that miR-98 may play a vital role in regulating macrophage polarization, thus suppressing the capacity of TAMs to promote invasion and EMT of HCC. Our study validated the vital role of miR-98-mediated macrophage polarization in $\mathrm{HCC}$ progression. It is our expectation that additional target genes of miR-98, such as IL-10, LIN28B, HMGA2, will be identified in the future. Our results suggest that miR-98 is a promising modulator for macrophage polarization and may become a promising therapeutic target for HCC treatment.

\section{Additional file}

Additional file 1: Figure S1. The differentiation proportion of different types of macrophages. (A) Human monocytes were isolated from PBMCs by sorting with anti-CD14 magnetic beads. Macrophages were prepared from these monocytes by culture for 7 days in RPMI 1640 medium

containing $10 \% \mathrm{FBS}$ in the presence of $50 \mathrm{ng} / \mathrm{ml}$ M-CSF. Flow cytometry revealed that the purified cells were $>95 \% \mathrm{CD} 14^{+}$cells. (B) To obtain M0 cells, CD $14^{+}$monocytes were treated with serum-free medium for $48 \mathrm{~h}$. To polarize M1 macrophages, macrophages were stimulated overnight with $100 \mathrm{ng} / \mathrm{ml}$ LPS, and $100 \mathrm{ng} / \mathrm{ml}$ IFN- $\gamma$. To polarize M2 macrophages, macrophages were stimulated with overnight with $20 \mathrm{ng} / \mathrm{ml} \mathrm{IL-4}$. The differentiation proportion of M0 (CD16/23-CD206), M1 (CD16/23+) and M2 $\left(\mathrm{CD}^{206^{+}}\right)$macrophages detected by flow cytometry was $87 \%, 89 \%$ and $96 \%$, respectively.

\section{Abbreviations}

ELISA: enzyme-linked immunosorbent assay; EMT: epithelial-mesenchymal transition; HCC: hepatocellular carcinoma; IL-1 $\beta$ : interleukin-1 $\beta$; miRNAs: microRNAs; PBMCs: peripheral blood mononuclear cells; TAMs: tumor-associated macrophages; TGF- $\beta$ : transforming growth factor- $\beta$; Th: Thelper; TNF-a: tumor necrosis factor-a.

\section{Authors' contributions}

LL designed the study; LL, PS and CZ performed the experiments; ZL and KC analyzed the data; WZ contributed analytical tools; WZ drafted the manuscript. All authors read and approved the final manuscript.

\section{Author details}

${ }^{1}$ Department of Hepatobiliary Surgery, Shandong Cancer Hospital Affiliated to Shandong University, Shandong Academy of Medical Sciences, Jinan 250117, Shandong, China. ${ }^{2}$ Department of Hepatobiliary and Pancreatic Surgery, Xuzhou Cancer Hospital, No. 131 Huancheng Road, Gulou District, Xuzhou 221000, Jiangsu, China.

\section{Acknowledgements}

Not applicable.

\section{Competing interests}

The authors declare that they have no competing interests.

\section{Availability of data and materials}

The datasets used and/or analysed during the current study are available from the corresponding author on reasonable request.

\section{Consent for publication}

Written informed consent was obtained from all donors.

Ethics approval and consent to participate

The study was approved by the Ethics Committee of Shandong Cancer Hospital Affiliated to Shandong University.

\section{Funding}

No funding was received.

\section{Publisher's Note}

Springer Nature remains neutral with regard to jurisdictional claims in published maps and institutional affiliations. 
Received: 4 January 2018 Accepted: 22 June 2018

Published online: 06 July 2018

\section{References}

1. Siegel RL, Fedewa SA, Miller KD, Goding-Sauer A, Pinheiro PS, MartinezTyson D, Jemal A. Cancer statistics for Hispanics/Latinos, 2015. Ca A Cancer J Clin. 2012;65(5):283-98.

2. Torre LA, Bray F, Siegel RL, Ferlay J, Lortettieulent J, Jemal A. Global cancer statistics, 2012. Ca A Cancer J Clin. 2015;65(2):87.

3. Wang $Z$, Lu X, Hu Y, Huang Y, Zhang Y, Zheng X, Wang S, Wang Y, Yu $Y$, Meng Z. miRNA let-7b modulates macrophage polarization and enhances tumor-associated macrophages to promote angiogenesis and mobility in prostate cancer. Sci Rep. 2016;6:25602.

4. Capece D, Fischietti M, Verzella D, Gaggiano A, Cicciarelli G, Tessitore A Zazzeroni F, Alesse E. The inflammatory microenvironment in hepatocellular carcinoma: a pivotal role for tumor-associated macrophages. Biomed Res Int. 2012;2013(1):187204.

5. Mcwhorter FY, Davis CT, Liu WF. Physical and mechanical regulation of macrophage phenotype and function. Cell Mol Life Sci. 2015;72(7):1303.

6. Sica A. Macrophages, innate immunity and cancer: balance, tolerance, and diversity. Curr Opin Immunol. 2010;22(2):231-7.

7. Murray PJ, Wynn TA. Protective and pathogenic functions of macrophage subsets. Nat Rev Immunol. 2011;11(11):723.

8. Sica A, Schioppa T, Mantovani A, Allavena P. Tumour-associated macrophages are a distinct $\mathrm{M} 2$ polarised population promoting tumour progression: potential targets of anti-cancer therapy. Eur J Cancer. 2006;42(6):717-27.

9. Solinas G, Schiarea S, Liguori M, Fabbri M, Pesce S, Zammataro L, Pasqualini F, Nebuloni M, Chiabrando C, Mantovani A. Tumor-Conditioned macrophages secrete migration-stimulating factor: a new marker for M2-polarization, influencing tumor cell motility. J Immunol. 2010;185(1):642-52.

10. Takayama H, Nishimura K, Tsujimura A, Nakai Y, Nakayama M, Aozasa K, Okuyama A, Nonomura N. Increased infiltration of tumor associated macrophages is associated with poor prognosis of bladder carcinoma in situ after intravesical bacillus Calmette-Guerin instillation. J Urol. 2009;179(4):1894-900.

11. Bacman D, Merkel S, Croner R, Papadopoulos T, Brueckl W, Dimmler A. TGF-beta receptor 2 downregulation in tumour-associated stroma worsens prognosis and high-grade tumours show more tumour-associated macrophages and lower TGF-beta1 expression in colon carcinoma: a retrospective study. BMC Cancer. 2007;7(1):156

12. Zhu XD, Zhang JB, Zhuang PY, Zhu HG, Zhang W, Xiong YQ, Wu WZ, Wang L, Tang ZY, Sun HC. High expression of macrophage colonystimulating factor in peritumoral liver tissue is associated with poor survival after curative resection of hepatocellular carcinoma. J Clin Oncol. 2008;26(16):2707-16.

13. Rizos E, Siafakas N, Katsantoni E, Skourti E, Salpeas V, Rizos I, Tsoporis JN, Kastania A, Filippopoulou A, Xiros N, et al. Let-7, mir-98 and mir-183 as biomarkers for cancer and schizophrenia [corrected]. PLOS ONE. 2015;10(4):e0123522.

14. Wendler A, Keller D, Albrecht C, Peluso JJ, Wehling M. Involvement of let-7/miR-98 microRNAs in the regulation of progesterone receptor membrane component 1 expression in ovarian cancer cells. Oncol Rep. 2011;25(1):273-9.

15. Zhang JJ, Chen JT, Hua L, Yao KH, Wang CY. miR-98 inhibits hepatocellular carcinoma cell proliferation via targeting EZH2 and suppressing Wnt/ beta-catenin signaling pathway. Biomed Pharmacother. 2017;85:472-8.
16. Wang YC, Chen YL, Yuan RH, Pan HW, Yang WC, Hsu HC, Jeng YM. Lin-28B expression promotes transformation and invasion in human hepatocellular carcinoma. Carcinogenesis. 2010;31(9):1516-22.

17. Zhou W, Zou B, Liu L, Cui K, Gao J, Yuan S, Cong N. MicroRNA-98 acts as a tumor suppressor in hepatocellular carcinoma via targeting SALL4. Oncotarget. 2016;7(45):74059-73.

18. Kaczmarek M, Nowicka A, Kozlowska M, Zurawski J, Batura-Gabryel H, Sikora J. Evaluation of the phenotype pattern of macrophages isolated from malignant and non-malignant pleural effusions. Tumour Biol. 2011;32(6):1123-32

19. Sakai Y, Honda M, Fujinaga H, Tatsumi I, Mizukoshi E, Nakamoto Y, Kaneko S. Common transcriptional signature of tumor-infiltrating mononuclear inflammatory cells and peripheral blood mononuclear cells in hepatocellular carcinoma patients. Cancer Res. 2008;68(24):10267-79.

20. Budhu A, Forgues M, Ye QH, Jia HL, He P, Zanetti KA, Kammula US, Chen Y, Qin LX, Tang ZY. Prediction of venous metastases, recurrence, and prognosis in hepatocellular carcinoma based on a unique immune response signature of the liver microenvironment. Cancer Cell. 2006;10(2):99.

21. Li YW, Qiu SJ, Fan J, Gao Q, Zhou J, Xiao YS, Xu Y, Wang XY, Sun J, Huang $\mathrm{XW}$. Tumor-infiltrating macrophages can predict favorable prognosis in hepatocellular carcinoma after resection. J Cancer Res Clin Oncol. 2009;135(3):439.

22. Chew V, Tow C, Teo M, Wong HL, Chan J, Gehring A, Loh M, Bolze A, Quek R, Lee VK. Inflammatory tumour microenvironment is associated with superior survival in hepatocellular carcinoma patients. J Hepatol. 2010;52(3):370.

23. Chaudhuri AA, So AY, Sinha N, Gibson WS, Taganov KD, O'Connell RM, Baltimore D. MicroRNA-125b potentiates macrophage activation. J Immunol. 2011:187(10):5062-8.

24. Androulidaki A, lliopoulos D, Arranz A, Doxaki C, Schworer S, Zacharioudaki V, Margioris AN, Tsichlis PN, Tsatsanis C. The kinase Akt1 controls macrophage response to lipopolysaccharide by regulating microRNAs. Immunity. 2009;31(2):220.

25. Kumar M, Sahu SK, Kumar R, Subuddhi A, Maji RK, Jana K, Gupta P, Raffetseder J, Lerm M, Ghosh Z. MicroRNA let-7 Modulates the immune response to Mycobacterium tuberculosis infection via control of A20, an inhibitor of the NF-kB pathway. Cell Host Microb. 2015;17(3):345-56.

26. Yu D, Yang L, Lv H, Zhou S, Zhen S, Min W. miR-98 suppresses tumor cel growth and metastasis by targeting IGF1R in oral squamous cell carcinoma. Int J Clin Exp Pathol. 2015;8(10):12252.

27. Ran N, Huang Y, Jing W. miR-98 targets ITGB3 to inhibit proliferation, migration, and invasion of non-small-cell lung cancer. Oncotargets Ther. 2015;8(default):2689-97.

28. Li F, Li X, Qiao L, Shi F, Liu W, Li Y, Dang Y, Gu W, Wang X, Liu W. miR-98 suppresses melanoma metastasis through a negative feedback loop with its target gene IL-6. Exp Mol Med. 2014;46(10):e116.

29. Fan YH, Ye MH, Wu L, Lv SG, Wu MJ, Xiao B, Liao CC, Ji QK, Chai Y, Zhu XG. Overexpression of miR-98 inhibits cell invasion in glioma cell lines via downregulation of IKKE. Eur Rev Med Pharmacol Sci. 2015;19(19):3593.

30. Lin X, Chen L, Yao Y, Zhao R, Cui X, Chen J, Hou K, Zhang M, Su F, Chen J, et al. CCL18-mediated down-regulation of miR98 and miR27b promotes breast cancer metastasis. Oncotarget. 2015;6(24):20485-99.

31. Shin HD, Park BL, Kim LH, Jung JH, Kim JY, Yoon JH, Kim YJ, Lee HS. Interleukin 10 haplotype associated with increased risk of hepatocellular carcinoma. Hum Mol Genet. 2003:12(8):901-6. 